\title{
Editorial
}

\section{Machine Learning and Visual Computing}

\author{
Lei Zhang, ${ }^{1}$ Yu Cao, ${ }^{2}$ Fei Yang, ${ }^{3}$ and Qiushi Zhao ${ }^{4}$ \\ ${ }^{1}$ Temple University, Philadelphia, PA, USA \\ ${ }^{2}$ Medical Sieve Radiology Grand Challenge, IBM Research, San Jose, CA, USA \\ ${ }^{3}$ Shandong University, Weihai, China \\ ${ }^{4}$ Harbin University of Science and Technology, Harbin, China \\ Correspondence should be addressed to Lei Zhang; cszhanglei@gmail.com
}

Received 29 January 2017; Accepted 29 January 2017; Published 19 March 2017

Copyright ( $\odot 2017$ Lei Zhang et al. This is an open access article distributed under the Creative Commons Attribution License, which permits unrestricted use, distribution, and reproduction in any medium, provided the original work is properly cited.

With the fast development of information science, information contained in big data has raised the interest of researchers from many different disciplines. Extracting and exploring the information from big datasets are essential to applying computational intelligence and soft computing to natural and social sciences. Recent advances of machine learning (especially, deep learning) make it possible to gain insight into big data and extract meaningful information, which has accelerated the progression of computational intelligence. Computer vision techniques contribute to understanding image and high-dimensional data from the real world to produce numerical or symbolic information. Visualization methods provide various ways to demonstrate information from complex datasets. Both computer vision techniques and visualization methods can be utilized to visually demonstrate the extracted information from datasets.

This special issue is dedicated to latest developments in machine learning and visual computing. Five articles from researchers around the world contribute to further steps into the theories and applications of machine learning and visual computing. The special issue covers a widespread research topics, from theoretical investigations to real-world applications, and comprises articles in active research areas like face classification, action recognition, and deep learning.

In the applications of computer vision techniques, $\mathrm{M}$. Ngadi et al. introduce a practical face detection method by using the Local Binary Patterns (LBP) for the feature extraction and a novel Neighboring Support Vector Classifier (NSVC) for classification. The experimental results on different natural images show that the proposed method can get promising results within a short detection time. G. Zhang et al. provide a novel person reidentification method which leverages the clothing features in real-life videos.

Toward improving machine learning algorithms based on theoretical finding, B. $\mathrm{Zu}$ et al. propose a LowRank Kernel-based Semi-supervised Discriminant Analysis (LRKSDA). Extensive experiments on public databases show that LRKSDA can outperform other related Kernel Semisupervised Discriminant Analysis methods. X. Li et al. propose an action recognition method by mining key skeleton poses with latent support vector machine (latent SVM). The detailed experimental results on three benchmark action datasets demonstrate that the proposed approach achieves superior performance to the state-of-the-art skeleton-based action recognition methods.

This special issue includes one review paper. The work by $\mathrm{D}$. Xie et al. provides a survey of deep learning. The authors not only reviewed typical deep learning algorithms in computer vision and signal processing but also provided detailed information on how to apply different deep learning techniques to specific areas such as road crack detection and fault diagnosis.

\section{Acknowledgments}

We would like to thank all the authors and reviewers for their great contributions to this special issue.

Lei Zhang Yu Cao

Fei Yang

Qiushi Zhao 

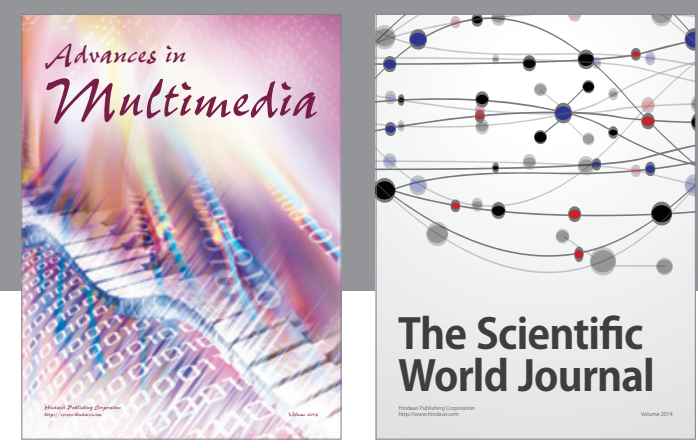

The Scientific World Journal
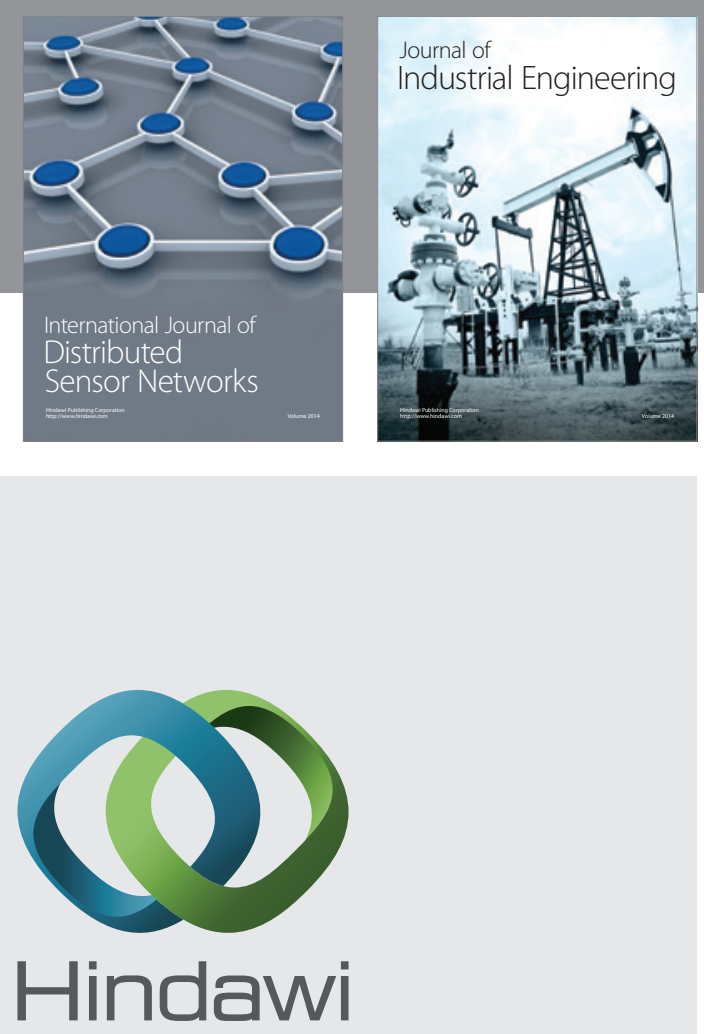

Submit your manuscripts at

https://www.hindawi.com

\section{Computer Networks} and Communications
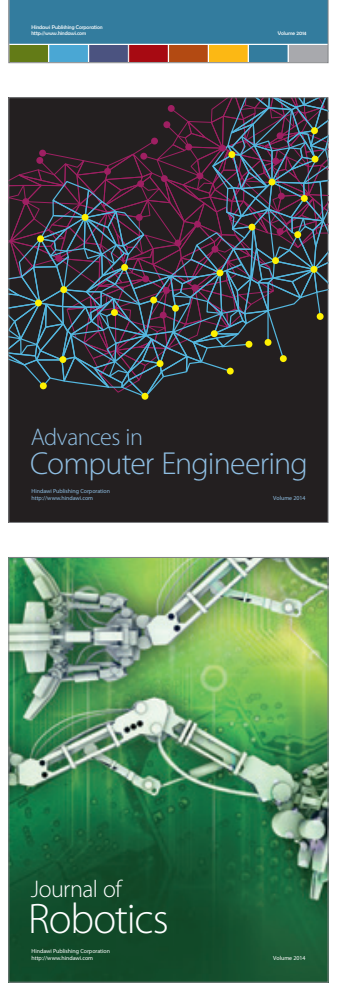
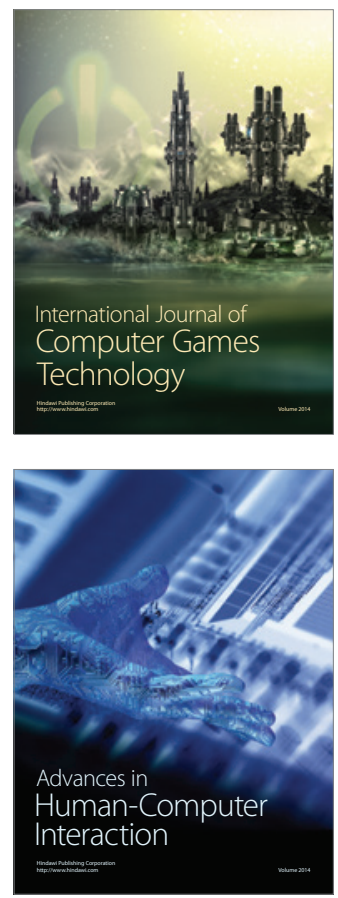
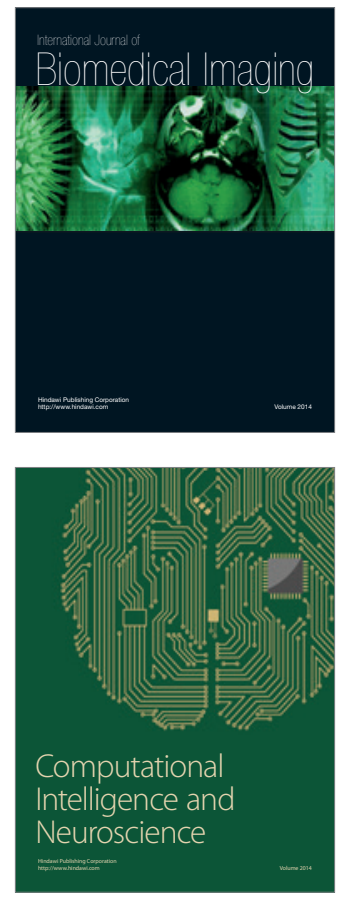
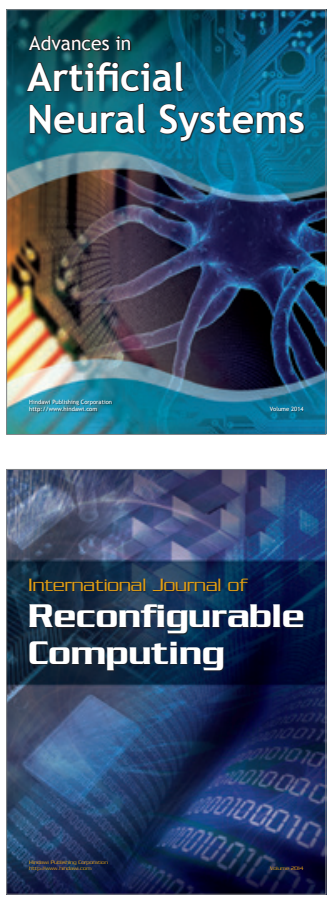
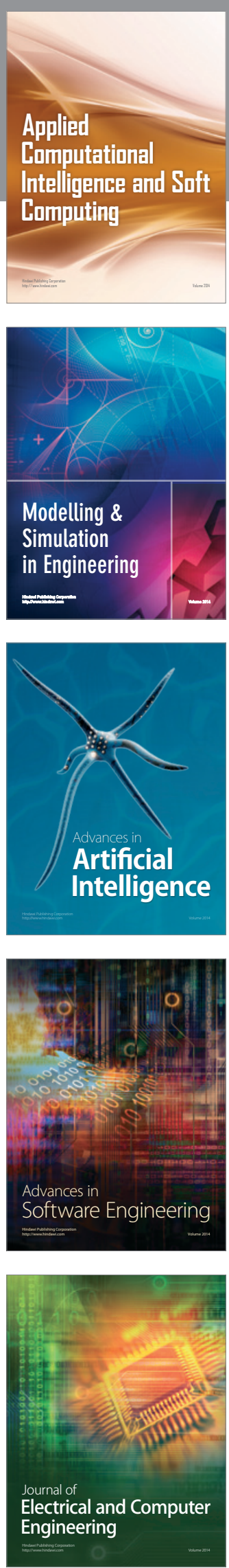